\title{
BMJ Open What is the best analgesic option for patients presenting with renal colic to the emergency department? Protocol for a systematic review and meta-analysis
}

\author{
Sameer A. Pathan, ${ }^{1,2,3}$ Biswadev Mitra, ${ }^{2,3,4}$ Lorena Romero, ${ }^{5}$ Peter A. Cameron ${ }^{2,3,4}$
}

To cite: Pathan SA., Mitra B, Romero $\mathrm{L}$, et al. What is the best analgesic option for patients presenting with renal colic to the emergency department? Protocol for a systematic review and meta-analysis. BMJ Open 2017;7:e015002. doi:10.1136/ bmjopen-2016-015002

- Additional material is published online only. To view please visit the journal online (http://dx.doi.org/10.1136/ bmjopen-2016-015002)

Received 1 November 2016 Revised 6 March 2017 Accepted 21 March 2017

\section{CrossMark}

'Emergency Department, Hamad General Hospital, Hamad Medical Corporation, Doha, Qatar

${ }^{2}$ Department of Epidemiology \& Preventive Medicine, Monash University, Melbourne, Australia ${ }^{3}$ National Trauma Research Institute, The Alfred Hospital, Melbourne, Australia

${ }^{4}$ Emergency \& Trauma Centre, The Alfred Hospital, Melbourne, Australia

${ }^{5}$ The lan Potter Library, The Alfred, Melbourne, Victoria, Australia

Correspondence to Dr Sameer A. Pathan; sameer.pathan@monash.edu

\section{ABSTRACT}

Introduction Patients with renal colic present to the emergency department in excruciating pain. There is variability in practice regarding the choice of initial analgesic to be used in renal colic. The aim of this article is to outline the protocol for review of the efficacy and safety of non-steroidal anti-inflammatory drugs (NSAIDs), opioids and paracetamol use in renal colic pain management. Methods and analysis This is the protocol for a systematic review, comparing efficacy of NSAIDs, opioids and paracetamol in renal colic studied under randomised controlled trial (RCT) design. This protocol reporting is based on the PRISMA-P recommendations (PRISMA-Pchecklist). We will conduct a comprehensive literature search for both peer-reviewed and grey literature published until 18 December 2016. Using a predefined search strategy, MEDLINE, Embase and Cochrane Central Register of Controlled Trials will be searched. Additional searches will include WHO International Clinical Trials Registry Platform, abstract list of relevant major conferences and the reference lists of relevant publications. Two authors will independently screen and identify the studies to be included. The RCT comparing NSAIDs versus opioids or paracetamol will be included in the review, if the age of participants in the study was $>16$ years and they presented with moderate to severe renal colic. Any disagreement between the screening authors will be resolved through discussion and reaching consensus; if not, a third reviewer will arbitrate. Quantitative data from homogeneous studies will be pooled in the meta-analysis using RevMan V.5.3 software. The findings of this review will be presented according to the guidelines in the Cochrane Handbook of Systematic Reviews of Interventions and the Preferred Reporting Items for Systematic Review and Meta-Analysis statement. Ethics and dissemination Formal ethics approval is not required, as primary data will not be collected. We plan to publish the result of this review in a peer-reviewed journal.

\section{INTRODUCTION}

Kidney stones are common in the 'stone belt' region, which extends over America (Southeast), Africa (North), Middle East Asia (Southeast), and Australia (Northeast). ${ }^{1}$ Globally, the lifetime prevalence of stone disease is $10 \%-15 \%$ and accounts for

\section{Strengths and limitations of this study}

This is the protocol for a systematic review and meta-analysis of randomised controlled trials

- We plan to follow the Preferred Reporting Items for Systematic review and Meta-Analysis Protocols, Grading of Recommendations Assessment, Development and Evaluation framework, and Cochrane tools for assessing the risk of bias.

- We anticipate difficulty in pooling data due to heterogeneity among the published research.

- It will provide robust evidence in deciding superiority among commonly used analgesics, and help to improve guidance for protocolised analgesia in renal colic.

millions of patient visits to emergency departments (ED) or outpatient clinics. ${ }^{12}$ The acute painful presentation is commonly known as renal colic, and movement of stone in the urinary tract produces this excruciating pain. The National Health Service, England statistics for year 2012-2013 estimated the cost for renal colic at nearly $£ 20$ million, where median patient stay in the hospital was 1 day. ${ }^{3}$ In the management of renal colic, one of the priorities in the ED is to provide quick, safe and effective analgesia.

The most commonly prescribed analgesics in renal colic are non-steroidal anti-inflammatory drugs (NSAIDs), opioids and paracetamol. ${ }^{45}$ The important factors in the selection of first-line analgesia in the ED are efficacy, safety, the ease of rapid administration, and the logistics involved. Effective ongoing analgesia can be practically challenging to deliver in an ED with a diverse population and a high volume of patients being managed concurrently. ${ }^{6}$ A previously published meta-analysis comparing NSAIDs with opioids suggested NSAIDs were better than opioids in terms of requiring less rescue analgesia and having fewer side 
effect. However, it did not establish superior efficacy of either drug group. ${ }^{57}$ Although the European Association of Urology guidelines on urolithiasis recommends NSAIDs as the first choice, the use of intravenous opioids as the first-line analgesic in renal colic continues to be a common practice in many developed countries. ${ }^{8-12}$ However, the logistical delay involved in intravenous administrations, dose-dependent side effects, need for titrating dosage and overly bureaucratic restrictions are some of the challenges associated with the intravenous opioid use as the first-line analgesic in the busy ED. ${ }^{13-15}$ Routine use of NSAIDs has been limited because of the fear of gastrointestinal (GI) and renal complications. In addition, there has been undue emphasis placed on the possibility of abscess and muscle necrosis secondary to intramuscular injection, given the extremely low level of documented cases.

The obvious limitations of previous studies and systematic reviews may partly explain the continued clinical orthodoxy of intravenous opioid use as the first analgesic in many settings. First, this review was conducted and published in 2004 and the studies included were published between 1982 and $1999 .{ }^{5}$ In the last 15 years, newer, well-powered, pragmatic clinical trials have been published with clinically relevant outcomes in renal colic management. Second, most studies in the review only included patients who had confirmed renal calculi on subsequent testing. This may limit the applicability of evidence in routine clinical practice where patients are treated with a clinical picture of renal colic well before any imaging can be performed. Third, significant heterogeneity between the studies included did not allow pooled analysis to test the superiority of a drug based on efficacy. ${ }^{45}$ A pooled analysis of NSAIDs other than ketorolac in the review showed a pain reduction of $4.6 \mathrm{~mm}$ (on visual analogue scale $0-100 \mathrm{~mm}$ ) only, which is minimal compared with the newer trial results. ${ }^{15}$ Fourthly, 12 of the 20 included trials used pethidine as their opioid arm, which is not a commonly used opioid in current practice. ${ }^{5}$ Lastly, studies available to include at the time of review lacked consistent reporting of serious adverse events such as renal failure and GI bleeding, limiting comparability.

There is ongoing controversy regarding the superiority of any of the commonly used analgesics in terms of efficacy, optimum dosing and route of administration. Therefore, we aim to examine the efficacy and safety of NSAIDs, opioids and paracetamol use in renal colic pain management.

\section{METHODS AND DESIGN}

\section{Types of participants}

The systematic review will only include studies involving adult patients (age $>16$ years) with a clinical diagnosis of acute renal colic (pain less than 12 hours) and moderate to severe pain severity. If a study reports data on both adult and paediatric populations, we will only include the data if the mean age of patients is over 18. The data from mixed population studies will be highlighted when reporting final results.

\section{Type of studies}

Only randomised controlled trials comparing NSAIDs versus opioids or NSAIDs versus paracetamol will be included in the review. There will be no language restriction to conduct primary search. If the language used to write the article is other than English, we will use a professional translator to translate the text in English.

\section{Types of interventions}

The studies will be reviewed if interventions include the following:

- NSAIDs versus opioids in any dose, by any route in any setting, used for pain relief in acute renal colic episode will be eligible.

- NSAIDs versus paracetamol (acetaminophen) in any dose, by any route in any setting, used for pain relief in acute renal colic episode will be eligible.

NSAIDs included will be salicylates, propionic acid derivatives, acetic acid derivatives, enolic acid derivatives, fenamates, selective Cox-2 inhibitors and sulfonamides.

\section{Types of outcome measures}

Studies with at least one of the following outcomes will be included:

- outcome measured using a validated pain scale

- difference in pain score at $30 \mathrm{~min}$

- difference in the proportion of patients with pain relief at $30 \mathrm{~min}$

- need for rescue analgesia

- adverse events as reported

- major adverse event (eg, GI bleeding and renal complications, complications at the intramuscular injection site).

\section{Information sources}

The search will not be restricted by language or date of publication to avoid publication or retrieval biases. We will search the following databases and sources for relevant studies:

- Cochrane Central Register of Controlled Trials (CENTRAL)

- Cochrane Renal Group Specialised Register for randomised controlled trials

- Ovid MEDLINE(R) 1946 to Present with Daily Update

- Ovid MEDLINE(R) In-Process \& Other Non-Indexed Citations

- Ovid MEDLINE (R) Epub Ahead of Print

- Embase Classic+ Embase 1947 to 2016 September

- WHO International Clinical Trials Registry Platform (http://apps.who.int/trialsearch/)

- Reference lists of nephrology, urology and emergency medicine textbooks, previously published review articles, and relevant trials 
- Abstract list of the major nephrology, urology and emergency medicine conferences

- Correspondence documents seeking information about unpublished or incomplete trials from the investigators known to be involved in previous trials.

The initial search strategy was developed in the Ovid MEDLINE database using subject heading and free-text words. The relevant subclasses of NSAIDs and commercial names for the commonly used drugs were searched through Google and used in the free-text search. We also compared the search strategies used for the previous two Cochrane reviews ${ }^{45}$ and modified our strategy if any important terms were found missing. Drugs such as phenylbutazone, aminophenazone (or aminopyrine) and ampyrone were excluded as these drugs have a risk of agranulocytosis and are no longer used as routine analgesics.

\section{Search strategy}

We will perform the first search on MEDLINE, Embase and Cochrane CENTRAL via Ovid. The detailed search strategy is attached as online supplementary appendix A. The second search will be performed for ongoing clinical trials on WHO International Clinical Trial Registry Platform. Finally, a manual search will be conducted in relevant key journals, conference abstracts, textbook chapters and the bibliography of included articles. Outcome of each database search will be individually imported into Endnote X7 (Thomson Reuters) reference manager. After this, using Endnote, the references will be combined and duplications will be removed. The record of total search results retrieved and screened will be kept and reported using the Preferred Reporting Items for Systematic Review and Meta-Analysis guidelines.

\section{Selection process}

Two authors will independently screen the titles and abstracts of de-duplicated results to identify potentially eligible studies. These studies then will be further reviewed independently, going through the full text, to confirm the inclusion criteria. Any disagreement will be resolved through discussion or by consulting a third review author. Agreement on independent inclusion of titles, abstract or full text will be quantified using K statistics. Reasons for excluding potentially eligible studies will be recorded and reported as supplementary to the main review.

\section{Data collection process}

Two authors will independently extract the data using Cochrane Collaboration Review Manager statistical software (RevMan V.5.3) (http://ims.cochrane.org/ RevMan) and a prepiloted Microsoft Excel sheet. Before starting the review, calibration exercises will be conducted among the reviewers to ensure consistency. Discrepancies between the data extraction will be resolved by discussion and reaching a consensus. If needed a third reviewer will be contacted to reach a decision.

\section{Data items}

Data will be collected on the following data points:

1. Research information: the first author, the site where the study was conducted, year of publication, research design (randomisation and concealed allocation) and the sample size.

2. Characteristics of the study subjects: Age, sex, numbers in each group, inclusion and exclusion of criteria of individual study, pain scores at the time of randomisation, and diagnostic confirmation of renal colic.

3. Information on intervention and comparison arms: number of groups, intervention and comparator(s) (drug, dose and route), and the information about blinding (treating person, assessor and patient). Information about exclusion after randomisation will also be recorded (intention to treat).

\section{Outcomes and prioritisation}

To compare outcomes, identification of the definition used for the primary outcome, measuring tool used, change in pain scores, need for rescue analgesia and at what time, side effects, and follow-up (GI bleed or renal impairment) will be assessed. Notes important but not classified in the above category will be entered as a free text.

\section{Quality assessment of studies}

To assess the risk of bias, study data will be extracted using the Cochrane Collaboration tool for assessing the risk of bias (table 8.5.a in the Cochrane Handbook for Systematic Reviews of Interventions). This includes information gathering on the method of randomisation, allocation concealment, blinding of participants and investigators, blinding of the assessor, incomplete outcome data and other bias if any. Each domain will be rated as having low, unclear or high risk of bias.

\section{Missing data}

We will try to contact corresponding authors to request missing data if contacts are available. If this is not achieved, we will try to impute the missing information based on the information available in the manuscript or the protocol of the study. For continuous measures, missing SD will be imputed from available information such as SE, CI or $\mathrm{p}$ values. For a dichotomous outcome, proportion or percentages will be used to calculate the number of events/people assessed for that outcome. The results will be interpreted considering the impact of missing data.

\section{ANALYSIS}

Data synthesis

For dichotomous outcomes, such as more than or equal to $50 \%$ reduction in pain, need for rescue analgesia, or 
adverse events, a risk ratio with $95 \%$ CI will be reported. The studies where continuous scale of measure were used to assess the primary effect, such as patient-rated pain, difference in mean pain score, a mean difference (MD) will be reported. If different measurement scales were used, a standardised MD will be used to express the results. For the adverse effects we will calculate the risk difference with $95 \%$ CI. Skewed data and non-quantitative data will be presented descriptively.

Quantitative data will be pooled in the meta-analysis using RevMan V.5.3 software. Fixed-effect models or random-effect models will be used appropriately based on the heterogeneity observed among the studies included in the pooled analysis. Statistical heterogeneity in each model will be assessed using the $\chi^{2}$ test of heterogeneity and quantified using the Higgins' $\mathrm{I}^{2}$ statistics. If the heterogeneity is insignificant, the Mantel-Haenszel method will be used for the fixed-effect model. We will use the random-effects model if significant statistical heterogeneity $\left(\mathrm{I}^{2} \geq 50 \%\right.$ or $\left.\mathrm{p}<0.1\right)$ is present.

\section{Subgroup analysis}

We intend to perform the primary analysis based on the treatment groups such as NSAIDs versus opioids and NSAIDs versus paracetamol. We will also perform subgroup analysis based on the types of opioids or NSAIDs used, routes of administration and quality of the study. If heterogeneity is substantial, we will not perform a meta-analysis; a narrative, qualitative summary will be done and the information will be presented using text and tables.

\section{Meta-bias(es)}

To assess reporting bias among the included studies, where possible, we will determine if there was any deviation from the published protocol or information registered prior to conduct of the study. If a study was published after 1 July 2005, we will screen the Clinical Trial Register at the International Clinical Trials Registry Platform of the WHO (http:/ /apps.who.int/trialssearch). To assess the possibility of publication bias, we also plan to use a funnel plot if 10 or more studies are available for the meta-analysis.

\section{Assessing cumulative evidence}

We will assess the quality of body of evidence for each outcome according to the Grading of Recommendations Assessment, Development and Evaluation working group methodology.

\section{DISCUSSION}

Renal colic is a common cause of ED presentations and the excruciating pain demands effective analgesia to be administered in the shortest possible time. To minimise the delay in rapid administration of effective and safe analgesia, an evidence-based protocol is needed. Previous reviews were inconclusive in establishing superior efficacy to support first-line analgesia. It is important to use the first-line agent that is most effective, has the best safety profile, and is quick and easy to administer with a single rather than titrated first dose. Given that there have been significant publications since the last review on this topic, we believe that it is likely that improved guidance for protocolised first-line analgesia for renal colic can be given.

\section{Ethics and dissemination}

Formal ethics approval is not required, as primary data will not be collected. We plan to publish the result of this review in a peer-reviewed journal. We believe that the results of this review will provide robust evidence in deciding superiority among commonly used analgesics, and help to improve guidance for protocolised analgesia in renal colic.

Contributor SAP is the guarantor. SAP, BM and PC drafted protocol for the systematic review. SAP and LR developed the search strategy. All authors contributed to the development of the selection criteria, the risk of bias assessment strategy and data extraction criteria. All authors read, provided feedback and approved the final manuscript.

Competing interests None declared.

Patient consent Not obtained.

Provenance and peer review Not commissioned; externally peer reviewed.

Open Access This is an Open Access article distributed in accordance with the Creative Commons Attribution Non Commercial (CC BY-NC 4.0) license, which permits others to distribute, remix, adapt, build upon this work non-commercially, and license their derivative works on different terms, provided the original work is properly cited and the use is non-commercial. See: http://creativecommons.org/ licenses/by-nc/4.0/

(c) Article author(s) (or their employer(s) unless otherwise stated in the text of the article) 2017. All rights reserved. No commercial use is permitted unless otherwise expressly granted.

\section{REFERENCES}

1. Fisang C, Anding R, Müller SC, et al. Urolithiasis--an interdisciplinary diagnostic, therapeutic and secondary preventive challenge. Dtsch Arzteb/ Int 2015;112(6):83-91.

2. Ghani KR, Roghmann F, Sammon JD, et al. Emergency department visits in the United States for upper urinary tract stones: trends in hospitalization and charges. J Urol 2014;191(1):90-6.

3. Pickard R, Starr K, MacLennan G, et al. Use of drug therapy in the management of symptomatic ureteric stones in hospitalised adults: a multicentre, placebo-controlled, randomised controlled trial and cost-effectiveness analysis of a calcium channel blocker (nifedipine) and an alpha-blocker (tamsulosin) (the SUSPEND trial). Health Technol Assess 2015;19(63):1-171 vii-viii.

4. Afshar K, Jafari S, Marks AJ, et al. Nonsteroidal anti-inflammatory drugs (NSAIDs) and non-opioids for acute renal colic. Cochrane Database Syst Rev 2015;6:Cd006027.

5. Holdgate A, Pollock T. Nonsteroidal anti-inflammatory drugs (NSAIDs) versus opioids for acute renal colic. Cochrane Database Syst Rev 2015;18(2):Cd004137.

6. Pines JM, Hollander JE. Emergency department crowding is associated with poor care for patients with severe pain. Ann Emerg Med 2008;51(1):1-5.

7. Labrecque M, Dostaler LP, Rousselle R, et al. Efficacy of nonsteroidal anti-inflammatory drugs in the treatment of acute renal colic. A metaanalysis. Arch Intern Med 1994;154(12):1381-7.

8. Dave C, Shetty S, Faraj K, et al. Nephrolithiasis Treatment \& Management: webmd. 2016, http://emedicine.medscape.com/ article/437096-treatment (accessed 05 Feb 2017).

9. Ketabchi AA, Ebad-Zadeh MR, Parvaresh S, et al. Opium dependency in recurrent painful renal lithiasis colic. Addict Health 2012;4(1-2):73-8.

10. Worster A. In: Renal colic. Thomas SH, ed. Emergency Department Analgesia: an Evidence-Based Guide. New York: Cambridge University Press, 2008:359-62. 
11. Bounes V, Vallé B, Concina F, et al. Treatment of acute renal Colic in US and french EDs: simulated cases and real cases in acute pain management. Am J Emerg Med 2016;34(10):1955-8.

12. Türk $C$, Petrík $A$, Sarica $K$, et al. EAU guidelines on diagnosis and conservative management of Urolithiasis. Eur Urol 2016;69:468-74.

13. Manjiani D, Paul DB, Kunnumpurath $S$, et al. Availability and utilization of opioids for pain management: global issues. Ochsner $J$ 2014;14(2):208-15.
14. Berterame S, Erthal J, Thomas $\mathrm{J}$, et al. Use of and barriers to access to opioid analgesics: a worldwide, regional, and national study. Lancet 2016;387(10028):1644-56.

15. Pathan SA, Mitra B, Cameron PA. Titrated doses are optimal for opioids in pain trials - Authors' reply. Lancet 2016;388(10048):961-2. 